\title{
Dynamics of an antibiotic oligopeptide
}

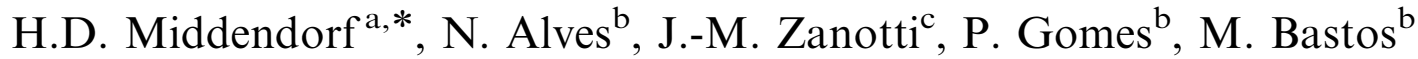

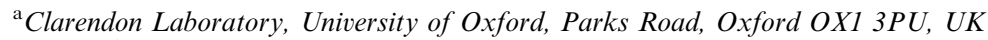 \\ ${ }^{\mathrm{b}} \mathrm{CIQ}$ (UP), Department of Chemistry, 4169-007 Porto, Portugal \\ ${ }^{\mathrm{c}}$ Laboratoire Léon Brillouin, CEA Saclay, 91191 Gif-sur-Yvette, France
}

\begin{abstract}
Neutron time-of-flight spectra were measured for an $\mathrm{H}_{2} \mathrm{O}$-hydrated and a nominally dry sample of a 15-residue antibacterial oligopeptide from 99 to $271 \mathrm{~K}$. Proton mobilities, quasielastic broadenings, and changes in low-frequency inelastic intensities characterise the evolution of the peptide energy landscape as a function of momentum transfer and temperature.

(C) 2006 Elsevier B.V. All rights reserved.
\end{abstract}

PACS: 29.30.Hs; 87.14.Ee; 64.70.Pf

Keywords: Inelastic scattering; Peptide dynamics; Proton mobilities; Boson peak

\section{Introduction}

The evolution of the energy landscape of proteins and peptides from low to near-ambient temperatures is a subject of central interest in current work on biodynamics $[1,2]$. Neutron techniques are uniquely suited to contribute data on the proton dynamics, either in the form of windowintegrated quasielastic intensities, $S_{\mathrm{qe}}(Q ; T)$, or as spectrally resolved dynamic structure factors, $S(Q, \omega ; T) \quad(\hbar Q=$ momentum transfer, $\hbar \omega=$ energy transfer). Most experiments on the low-temperature dynamics of proteins have aimed to derive mean-square proton amplitudes, $\left\langle u_{\mathrm{p}}^{2}\right\rangle$, from effective or modified Debye-Waller (DW) factors extracted from $S_{\mathrm{qe}}(Q ; T)$ functions measured at intermediate to high $Q$ between $\approx 30$ and $300 \mathrm{~K}$ [2]. In recent work, the emphasis has been increasingly on (i) examining the spectral changes responsible for deviations from idealised behaviour and (ii) bridging the interpretational gap between $S_{\mathrm{qe}}(Q ; T)$ studies of small biomolecular building blocks and those of proteins. In parallel with quasielastic neutron scattering (QENS) experiments on di- and tripeptides of biomedical interest [3,4], we have begun to study time-of-flight spectra from a larger peptide. Here, we

\footnotetext{
*Corresponding author. Tel.: + 441442 863828; fax: + 441865272400.

E-mail address: hdm01@isise.rl.ac.uk (H.D. Middendorf).
}

report initial results from an inelastic neutron scattering (INS) experiment on cecropin-mellitin, a biochemically and pharmacologically important 15 -residue oligopeptide.

\section{Materials and methods}

This hybrid oligopeptide, called CM15 in the following, is a prominent member of a family of eukaryotic antimicrobial peptides which are being studied intensely by a variety of techniques to optimise potency and specificity, focusing in particular on their membrane interactions [5,6]. CM15 is a hybrid comprising seven amino acid residues from cecro/pin A and eight from mellitin: CA(1-7)M(2-9), with sequence H-KWKLFKKIGAVLKVL-NH $\mathrm{N}_{2}$. A large sample $(350 \mathrm{mg})$ of CM15 was synthesised at Porto, and $\mathrm{H}_{2} \mathrm{O}$-hydrated to a specific hydration $h$ of $0.49(\mathrm{~g} / \mathrm{g})$. The nominally dry sample contained residual, structurally essential water at a level $h=0.068$, as determined by Karl Fischer titration. We used the spectrometer MIBEMOL at LLB (incident wavelength $5 \AA$, elastic $Q$-range $0.51-2.38 \AA^{-1}$ ) to record sets of neutron spectra over energy transfers $\hbar \omega$ from -2.3 to $\approx 200 \mathrm{meV}$ with a resolution of $0.195 \mathrm{meV}$ (FWHM) at $\hbar \omega=0$. Standard programs were employed to correct the data and to normalise them with respect to monitor counts and a vanadium run. 


\section{Results and discussion}

Data analysed are from three temperature series - (1) a set of spectra for $\mathrm{CM} 15 \cdot \mathrm{H}_{2} \mathrm{O}$ from long runs $(6-10 \mathrm{~h}$ each) at eight temperature points between 99 and $271 \mathrm{~K}$, (2) a continuous 8-h scan for $\mathrm{CM} 15 \cdot \mathrm{H}_{2} \mathrm{O}$ from 196 to $269 \mathrm{~K}$ during which spectra were taken every $7 \mathrm{~min}$, and (3) a set of 1-4h runs for the 'dry' CM15 sample at temperatures corresponding to, or very near, those of (1). It was not possible in this experiment, for technical reasons, to record spectra below $99 \mathrm{~K}$. The quality of the spectra from (1) is such that small inelastic intensity differences down to a few $10^{-4}$ of $S(Q, 0)$ are analysable with confidence. By contrast, individual spectra from the continuous $\mathrm{T}$-scan are statistically poor in the inelastic region proper, but their window-integrated quasielastic intensities are comparable in quality to data from scans using the backscattering spectrometer IN13 at ILL [2], providing $S_{\mathrm{qe}}(Q ; T)$ functions over a fine temperature grid in the region where the dynamics begins to be affected by softer modes and anharmonic interactions.

The quantity of immediate interest in experiments of this kind is $S_{\mathrm{qe}}(Q ; T)$. Since for hydrogenous biomolecules the scattering is incoherent to better than $95 \%, S_{\mathrm{qe}}(Q ; T)$ is essentially proportional to a proton-weighted DW factor in regions where the dynamic behaviour is adequately represented by a system of harmonic oscillators. Up to at least $150 \mathrm{~K}$, a m.-sq. displacement $\left\langle u_{\mathrm{p}}^{2}\right\rangle$ averaged over all protons can be derived from the slope of $\ln S_{\mathrm{qe}}(Q ; T)$ vs. $Q^{2}$ for $Q>1 \AA^{-1}$, and this $\left\langle u_{\mathrm{p}}^{2}\right\rangle$ increases essentially linearly with temperature (except at very low $T$, not covered in our experiment). At higher temperatures, usually around and above $200 \mathrm{~K}$, all hydrated biomolecules show significant nonlinear increases in $\left\langle u_{\mathrm{p}}^{2}\right\rangle$. These may be modelled analytically as products of effective DW factors and barrier-jump terms [3], as integrals over a distribution of $\left\langle u_{\mathrm{p}}^{2}\right\rangle$ values [7], or fitted by Plazcek-type expansions $\sim \exp \left(-\left\langle u_{\mathrm{p}}^{2}\right\rangle Q^{2}+c Q^{4}+\cdots\right)$.

A comprehensive analysis is not possible on the basis of the data we have collected so far, but it seems that in $\mathrm{CM} 15 \cdot \mathrm{H}_{2} \mathrm{O}$, we are observing three rather than two mobility regimes (Fig. 1) : first one up to $\approx 150 \mathrm{~K}$, then a 'ledge' region with a roughly linear dependence up to $\approx 235 \mathrm{~K}$, and finally a steep nonlinear rise similar to that in some globular proteins. Quantitative analysis of the latter two regions in terms of the models mentioned requires data up to at least $5 \AA^{-1}$ to allow extraction of parameters additional to the Gaussian DW term which dominates for $T<235 \mathrm{~K}$. Comparisons with high-resolution backscattering data for small proteins [1-3] show that overall the $\left\langle u_{\mathrm{p}}^{2}\right\rangle$ values for $\mathrm{H}_{2} \mathrm{O}$-hydrated $\mathrm{CM} 15$ are larger by factors between 1.1 and 1.3 , due in part to the wider proton time scale range sampled in our experiment (quasielastic window $\pm 0.2 \mathrm{meV}$ ), and in part to the fact that relative to tightly folded globular proteins (such as crambin [3] or BPTI), our hybrid oligopeptide is likely to retain more hydration-induced sidechain mobility at low temperatures

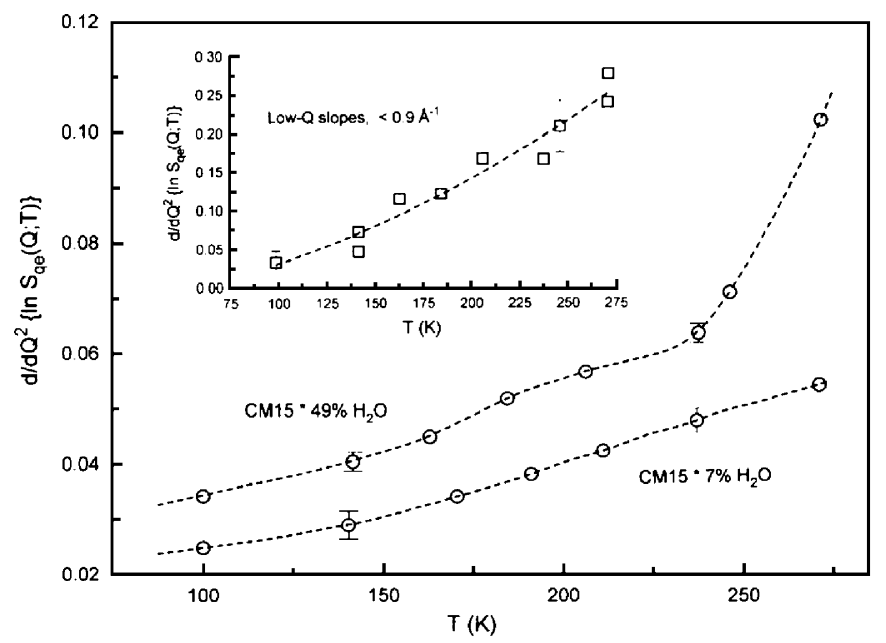

Fig. 1. Temperature dependence of $\left|d \ln S_{\mathrm{qe}}(Q ; T) \mathrm{d} Q^{2}\right|$ for hydrated and 'dry' CM15, as determined from $1.5<Q^{2}<4 \mathrm{~A}^{-2}$ (main graph) and for low $Q$ from $0.3<Q^{2}<0.85 \AA^{-2}$ (insert, hydrated CM15 only). In the harmonic and quasiharmonic regions $(<230 \mathrm{~K}$ for hydrated CM15) where the Gaussian (i.e. DW) component dominates, the ordinate may be identified with $\left\langle u_{\mathrm{p}}^{2}\right\rangle / 3$ and the resulting proton displacements $\left\langle u_{\mathrm{p}}\right\rangle$ range from 0.27 to $0.43 \AA$.

( 5 of the 15 amino acid residues are lysine, and there are 13 $\mathrm{CH}_{3}$ groups). This qualitative interpretation appears to be corroborated by the fact that we observe $\left\langle u_{\mathrm{p}}^{2}\right\rangle$ differences between the hydrated and the 'dry' sample that are larger than in crambin.

The $\mathrm{d}\left\{\ln S_{\mathrm{qe}}(Q ; T)\right\} / \mathrm{d} Q^{2}$ slopes reported in Fig. 1 (main graph) were derived from linear regression fits in the interval $1.5<Q^{2}<4 \AA^{-2}$. Between $\approx 0.9$ and $1.1 \AA^{-1}$, the behaviour of $S_{\mathrm{qe}}(Q ; T)$ is affected by uncertainties in the edge-on slab correction, but below $0.9 \AA^{-1}$, the temperature dependence of $\mathrm{d}\{\ln (Q ; T)\} / \mathrm{d} Q^{2}$ shows a systematic trend which differs significantly from the $Q^{2}>1.5 \AA^{-2}$ region, although it is less well defined statistically (Fig. 1, inset). The main feature here is the apparent absence of a 'knee' in the $220-240 \mathrm{~K}$ region, an observation suggesting substantially different proton dynamics over distances of $\approx 0.7-12 \AA$. Little work has been done as yet on the interpretation of biomolecular $S_{\mathrm{qe}}(Q ; T)$ data at low $Q$. This is a challenging preliminary result well worth following up in the future.

The levels of proton mobility inferred from $S_{\mathrm{qe}}(Q ; T)$ are reflected in the quasielastic broadening (Fig. 2). At the resolution of MIBEMOL for $\lambda_{0}=5 \AA$, the scattering around $\hbar \omega=0$ is effectively elastic until $\approx 230 \mathrm{~K}$ and gives rise to better defined quasielastic wings only towards $270 \mathrm{~K}$. The small broadening at $242 \mathrm{~K}$ seems to saturate for $Q \gg 1 \AA^{-1}$ near a level of $4 \mu \mathrm{eV}$, which on the basis of commonly used jump diffusion models corresponds to a characteristic time of about $150 \mathrm{ps}$. It will be of interest in future QENS experiments with $\mu \mathrm{eV}$ resolution to detect and quantify any broadening between 170 and $235 \mathrm{~K}$; this would aid greatly in the interpretation of the dynamics in the 'ledge' region of $\left\langle u_{\mathrm{p}}^{2}\right\rangle$. 


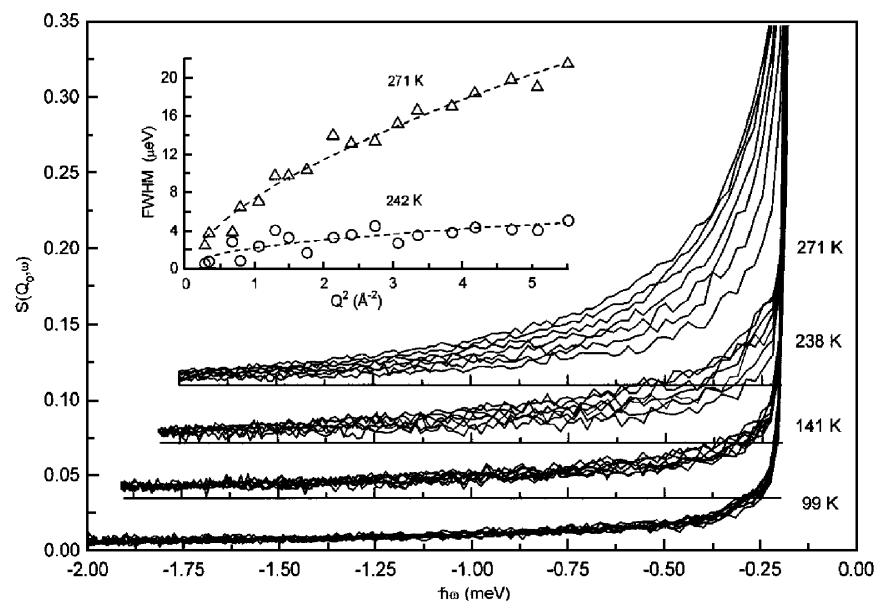

Fig. 2. Evolution of quasielastic intensities on the energy-loss side of spectra for $\mathrm{H}_{2} \mathrm{O}$-hydrated CM15. Every third spectrum from sets of 19 (binned down from 71 raw spectra, no smoothing) is shown; q.e. peak heights for the 238 and $271 \mathrm{~K}$ spectra are between 9 and 16. Inset: quasielastic broadenings (EISF model with single Lorentzian) for the two highest temperatures studied.

The temperature dependence of spectra in the $0.1-10 \mathrm{meV}$ region relates to a number of basic questions about specific relaxation processes known from homopolypeptides and the way in which they mix or couple in a more complex oligopeptide. A full discussion of the lowfrequency inelastic region is beyond the scope of this paper, but in view of the much debated (but rather enigmatic) 'Boson peak' observed in a variety of biomolecular systems, we have fitted Debye density of states $\sim \omega^{2}$ to appropriately reduced spectra. We find that for $T \lesssim 200 \mathrm{~K}$, the low-frequency region is well modelled by Debye curves, with a conspicuous band of 5-15\% 'excess' intensity extending from $\approx 2.2$ to $4.5 \mathrm{meV}$. As $T$ increases, the total 'excess' intensity decreases and the upper boundary shifts to slightly lower values (centroids shifting from 3.45 to $3.2 \mathrm{meV})$.

\section{Acknowledgment}

Experiments at LLB were supported by EU programmes NMI3 and FP6.

\section{References}

[1] J. Fitter, T. Gutberlet, J. Katsaras (Eds.), Neutrons in Biology, Springer, Berlin, 2005.

[2] F. Gabel, D. Bicout, U. Lehnert, M. Tehei, M. Weik, G. Zaccai, Q. Rev. Biophys. 35 (2002) 327.

[3] U.N. Wanderlingh, C. Corsaro, R.L. Hayward, M. Bée, H.D. Middendorf, Chem. Phys. 292 (2003) 445.

[4] M.T.F. Telling, C. Corsaro, U.N. Wander-lingh, H.D. Middendorf, Low-temperature Dynamics of Hydrated Glutathione, NIB 2005, Institut Laue-Langevin, Grenoble, 2005.

[5] K. Bhargava, J.B. Feix, Biophys. J. 86 (2004) 329.

[6] F. Abrunhosa, S. Faria, P. Gomes, I. Tomaz, J.C. Pessoa, D. Andreu, M. Bastos, J. Phys. Chem. B 109 (2005) 17311.

[7] H. Nakagawa, H. Kamikubo, I. Tsukushi, T. Kanaya, M. Kataoka, J. Phys. Soc. Japan 73 (2004) 491. 